This information is current as of April 26, 2023.

\title{
Acute Stroke Imaging: CT with CT Angiography and CT Perfusion before Management Decisions
}

A.J. Fox, S.P. Symons, P. Howard, R. Yeung and R.I. Aviv

AJNR Am J Neuroradiol 2012, 33 (5) 792-794

doi: https://doi.org/10.3174/ajnr.A3099

http://www.ajnr.org/content/33/5/792 
software of different vendors but also among different software versions from the same vendor, limiting their generalizability. This problem is one of standardization; effort is currently underway within the stroke community to address this. ${ }^{12}$ It is equally noteworthy, however, that for the purpose of selecting patients for endovascular stroke therapy, the correlation between CTP and DWI ischemic lesion volumes need not be perfect: CTP should only be able to accurately distinguish large $(>70-100 \mathrm{~mL})$ from small $(<70-100 \mathrm{~mL})$ admission ischemic lesion volumes. Preliminary data suggest that this is indeed the case. ${ }^{13}$

On the basis of these considerations, our neuroradiology section, in a consensus symposium led by Gil Gonzalez, developed the following acute stroke imaging algorithm delineating the imaging evaluation that we consider to be essential and sufficient for determining stroke treatment eligibility in our practice: ${ }^{6}$

1) Unenhanced head CT to exclude hemorrhage

2) Head and neck CTA, performed immediately following head CT (while the IV-tPA is being mixed, so as not to slow thrombolysis administration)

a) Axial, coronal, and sagittal thick-slab maximum intensity projections $(3-\mathrm{cm}$ section thickness at $0.5-\mathrm{cm}$ overlapping intervals) reviewed in real-time at the scanner console

b) If MR imaging is contraindicated and endovascular therapy will not immediately to be performed, CT perfusion imaging should be considered.

3) DWI

a) If large-vessel occlusion is present and infarct core is $<70-100 \mathrm{~mL}$, proceed immediately to endovascular treatment.

b) If the patient is not an endovascular candidate, MR perfusion imaging should be considered.

With regard to perfusion imaging, which is not required for either IV lysis or endovascular treatment selection, valid indications include the following: 1) excluding stroke mimics; 2) identifying high-risk patients following TIA; 3) specifying stroke subtype and hemodynamics; 4) clarifying/confirming the presence/site of vessel occlusion; 5) assessment of vasospasm; 6) determining the need for blood pressure management; 7) guiding disposition decisions, such as transfer to an intensive care unit; and 8) establishing prognosis, especially of large "malignant" perfusion patterns at early time points, for which the risks of treatment may outweigh the benefits (ie, too bad to treat). For these reasons, perfusion imaging is routine in our institutional acute stroke imaging algorithm, provided that definitive standard of care IV or endovascular reperfusion therapy will not be delayed.

\section{References}

1. Lyden PD. Advanced brain imaging studies should not be performed in patients with suspected stroke presenting within 4.5 hours of symptom onset. Stroke 2011;42:2668-69

2. Saver JL. Time is brain-quantified. Stroke. 2006;37:263-66

3. Maas MB, Lev MH, Ay H, et al. Collateral vessels on $\mathrm{CT}$ angiography predict outcome in acute ischemic stroke. Stroke. 2009;40:3001-05

4. Schellinger PD, Bryan RN, Caplan LR, et al. The role of diffusion and perfusion MRI for the diagnosis of acute ischemic stroke: report of the Therapeutics and Technology Assessment Subcommittee of the American Academy of Neurology. Neurology 2010;75:177-85

5. Yoo AJ, Verduzco LA, Schaefer PW, et al. MRI-based selection for intra-arterial stroke therapy: value of pretreatment diffusion-weighted imaging lesion volume in selecting patients with acute stroke who will benefit from early recanalization. Stroke 2009;40:2046-54

6. Yoo AJ, Pulli B, Gonzalez RG. Imaging-based treatment selection for intravenous and intra-arterial stroke therapies: a comprehensive review. Expert Rev Cardiovasc Ther 2011;9:857-76

7. Heiss WD, Sobesky J, Smekal U, et al. Probability of cortical infarction predicted by flumazenil binding and diffusion-weighted imaging signal intensity: a comparative positron emission tomography/magnetic resonance imaging study in early ischemic stroke. Stroke 2004;35:1892-98

8. Pulli B, Schaefer PW, Hakimelahi R, et al. Acute ischemic stroke: infarct core estimation on CT angiography source images depends on CT angiography protocol. Radiology 2011;262:593-604

9. Souza LC, Yoo AJ, Chaudry Z, et al. Malignant CTA collateral profile is highly specific for large admission DWI infarct core and poor outcome in acute stroke. AJNR Am J Neuroradiol 2012 March 1 [Epub ahead of print]

10. Kamalian S, Kamalian S, Maas MB, et al. CT cerebral blood flow maps optimally correlate with admission diffusion-weighted imaging in acute stroke but thresholds vary by postprocessing platform. Stroke 2011;42:1923-28

11. Campbell BC, Christensen S, Levi CR, et al. Cerebral blood flow is the optimal CT perfusion parameter for assessing infarct core. Stroke 2011;42:3435-40

12. Wintermark M, Albers GW, Alexandrov AV, et al. Acute stroke imaging research roadmap. AJNR Am J Neuroradiol 2008;29:e23-30

13. Souza LC, Lev MH, Franceschi AM, et al. Thresholded CTP maps can accurately determine infarct core when DWI is unavailable, and have similar specificity in identifying patients unlikely to benefit from thrombolysis. In: Proceedings of the 97th Scientific Assembly and Annual Meeting of the Radiological Society of North America, Chicago, Illinois. November 27-December 2, 2011

M.H. Lev

Massachusetts General Hospital Harvard Medical School

Boston, Massachusetts

http://dx.doi.org/10.3174/ajnr.A3098

\section{EDITORIAL}

\section{Acute Stroke Imaging: CT with CT Angiography and CT Perfusion before Management Decisions}

W ith less than stellar success of immediate intra-arterial therapy for acute stroke, $\mathrm{Cloft}^{1}$ mentions the need for "penumbra imaging" or advanced imaging to aid in management decisions. CT and MR approaches to such imaging are still not used by many interventionalists. There is more than just a side interest for acute stroke imaging protocols to be performed before such urgent management decisions. The goal of acute stroke therapy is the treatment of patients with ischemic brain to minimize infarct progression while not intervening when infarction is already complete.

For decades, imaging was performed to exclude other causes of stroke, parenchymal hematoma, mass, subdural hematoma, and seizure foci. Present day management decisions need imaging protocols with the following features: 1) demonstration of the intracranial arteries, extent of ischemia, and extent of completed infarction; 2) ability to be carried out promptly, conveniently, consistently, and accurately; and 3) immediate availability, facilitating management decisions for treatment (whether intra-arterial or intra-venous treatment).

Simple reliance on a NCCT and NIHSS threshold to inform clinicians of the presence and possible proximal location of a thrombus is not dependable for distinguishing ischemia from completed infarction. Although a higher NIHSS score may be seen with more proximal occlusions, a recent study of 
699 patients demonstrated that $55 \%$ of patients with significant occlusions had an NIHSS score of $\leq 10,{ }^{2}$ with a sensitivity for detection of a proximal occlusion in only $48 \%$ by using an NIHSS cutoff of $\geq 10 .{ }^{3}$ Similarly, high NIHSS scores can be seen without vessel occlusions in $\leq 30 \%$ of patients. ${ }^{4}$

Cloft ${ }^{1}$ states that penumbra imaging is still at the research stage without a consensus of approach. Consensus is not always the proper judge of research evidence. The value of CTP and CTA in conjunction with acute stroke imaging is widely reported. ${ }^{1,5-15}$ Informative structural and physiologic data can be obtained quickly for acute stroke presentations. Rapid acquisition is needed to meet the "Time is Brain" rally cry of acute stroke treatment.

We propose that acute CT protocols currently have a practical advantage over MR imaging protocols. Despite the uncontested sensitivity of DWI for core representation, primary MR imaging-based acute stroke protocols are rarely performed in tertiary institutions. Magnetic safety precautions, longer scanning duration, and concerns for managing potentially restless or unstable patients within the confined magnet environment limit MR imaging as a technique in the acute stroke setting. Time is expended on stringent safety checks, impractical for unaccompanied dysphasic patients, ironically necessitating CT head screening for orbit evaluation and a CT body scout for implant exclusion before MR imaging. These considerations and limited access to acute MR imaging in many centers render MR imaging impractical to direct immediate stroke management. Even expert sites in advanced MR stroke imaging begin with NCCT and commonly commence IV-rtPa on the basis of clinical presentation and NCCT alone before MR imaging. A protocol delaying thrombolysis after baseline NCCT pending DWI volume estimation amounts to a delay in time to treatment and undervalues the role of CT perfusion in infarct core estimation.

Acute CT stroke protocols (for our team, noncontrast CT, CTA arch to vertex, CTP, and postcontrast CT [PCCT]) need 2 minutes of scanning, little more than the time for noncontrast CT alone. The technical success of acute CT protocols requires organizing $\mathrm{CT}$ technologists to do efficient CTA and CTP postprocessing. Our team has $>20$ trained CT technologists able to perform and process acute stroke protocols 24/7 with standard CTA reformats as quick maximum-intensityprojection multiplanar reformations and CTP maps. Emerging data support CTP use to identify infarct core and at-risk ischemic tissue. Several challenges remain, including the optimal processing technique and determination of thresholds that best predict radiologic and clinical outcome. Yet, there is a convergence of data showing that both CBF and CBV thresholds demonstrate high correlation with DWI core,${ }^{16-18}$ while $\mathrm{CBF}$, time-to-maximum (T-max), and mean-transit-time thresholds correlate with penumbral tissue. ${ }^{16,19}$

A number of core surrogates facilitate interpretation of perfusion maps, including early ischemic changes on NCCT and hypoattenuation on PCCT. Ischemia is shown with CBF and CTA source images on modern CT scanners when CTA is performed first. ${ }^{9}$ Irrespective of precise tissue-specific thresholds, a large mismatch identifies candidates ideal for revascularization. ${ }^{20,21}$ Consideration of multiple available CT sequences increases confidence for correct stroke diagnosis among inexperienced readers ${ }^{22}$ and may facilitate identifica- tion of stroke mimics. ${ }^{23}$ The renal safety of iodinated contrast and radiation dose concerns are addressed in acute stroke CT. ${ }^{24-31}$ Last, CT-based protocols have cumulative data to widely apply to strokes, safely and cost-effectively. ${ }^{32,33}$

Neurointerventionalists are strongly motivated to treat occluded vessels, spurred on by case successes, despite lack of notable overall statistical success, as reviewed by Cloft. ${ }^{1}$ Stateof-the-art full stroke imaging seems key for high-level interventional techniques to more appropriately select candidates likely to improve with revascularization. Practical issues relating to expertise in image acquisition and postprocessing potentially may limit wide use for vascular and physiologic imaging. Incomplete imaging of patients with stroke can result in an inability to determine suitable candidates for intravenous thrombolytic or interventional treatments. Improved patient selection could optimize time-consuming, staff-intense, and expensive interventional neuroradiology normalized ratio procedures. This change would allow improved allocation of these services and avoid futile procedures that expose patients only to risk, without chance for benefit.

\section{References}

1. Cloft HJ. Death and destruction in the intra-arterial battle with acute ischemic stroke. AJNR Am J Neuroradiol 2011;32:1769-70

2. Derex L, Nighoghossian N, Hermier M, et al. Early detection of cerebral arterial occlusion on magnetic resonance angiography: predictive value of the baseline NIHSS score and impact on neurological outcome. Cerebrovasc Dis 2002;13:225-29

3. Maas MB, Furie KL, Lev MH, et al. National Institutes of Health Stroke Scale score is poorly predictive of proximal occlusion in acute cerebral ischemia. Stroke 2009;40:2988-93

4. Hacke W, Furlan AJ, Al-Rawi Y, et al. Intravenous desmoteplase in patients with acute ischaemic stroke selected by MRI perfusion-diffusion weighted imaging or perfusion CT (DIAS-2): a prospective, randomised, double-blind, placebo-controlled study. Lancet Neurol 2009;8:141-50

5. Lee TY, Murphy BD, Aviv RI, et al. Cerebral blood flow threshold of ischemic penumbra and infarct core in acute ischemic stroke: a systematic review. Stroke 2006;37:2201

6. Aviv RI, Mandelcorn J, Chakraborty S, et al. Alberta Stroke Program Early CT Scoring of CT perfusion in early stroke visualization and assessment. AJNR Am J Neuroradiol 2007;28:1975-80

7. Aviv RI, Shelef I, Malam S, et al. Early stroke detection and extent: impact of experience and the role of computed tomography angiography source images. Clin Radiol 2007;62:447-52

8. Tan IY, Demchuk A, Hopyan, et al. CT-angiography clot burden score and collateral score: correlation with clinical and radiological outcomes in acute middle cerebral artery territory infarct. AJNR Am J Neuroradiol 2009;30: 525-31

9. Sharma M, Fox AJ, Symons S, et al. CT angiographic source images: flow- or volume-related? AJNR Am J Neuroradiol 2011;32:359-64

10. Schaefer PW, Ozsunar Y, He J, et al. Assessing tissue viability with MR diffusion and perfusion imaging. AJNR Am J Neuroradiol 2003;24:436-43

11. Rohl L, Ostergaard L, Simonsen CZ, et al. Viability thresholds of ischemic penumbra of hyperacute stroke defined by perfusion-weighted MRI and apparent diffusion coefficient. Stroke 2001;32:1140-46

12. Kaufmann AM, Firlik AD, Fukui MB, et al. Ischemic core and penumbra in human stroke. Stroke 1999;30:93-99

13. Furlan M, Marchal G, Viader F, et al. Spontaneous neurological recovery after stroke and the fate of the ischemic penumbra. Ann Neurol 1996;40:216-26

14. Schlaug G, Benfield A, Baird AE, et al. The ischemic penumbra: operationally defined by diffusion and perfusion MRI. Neurology 1999;53:1528-37

15. Schaefer PW, Roccatagliata L, Ledezma C, et al. First-pass quantitative CT perfusion identifies thresholds for salvageable penumbra in acute stroke patients treated with intra-arterial therapy. AJNR Am J Neuroradiol 2006;27: $20-25$

16. Murphy BD, Fox AJ, Lee DH, et al. Identification of penumbra and infarct in acute ischemic stroke using computed tomography perfusion-derived blood flow and blood volume measurements. Stroke 2006;37:1771-77

17. Kamalian S, Kamalian S, Maas MB, et al. CT cerebral blood flow maps optimally correlate with admission diffusion-weighted imaging in acute stroke but thresholds vary by postprocessing platform. Stroke 2011;42:1923-28 
18. Campbell BC, Christensen S, Levi CR, et al. Cerebral blood flow is the optimal CT perfusion parameter for assessing infarct core. Stroke 2011;42:3435-40

19. Kamalian S, Kamalian S, Konstas A, et al. CT perfusion mean transit time maps optimally distinguish benign oligemia from true "at-risk" ischemic penumbra, but thresholds vary by postprocessing technique. AJNR Am J Neuroradiol 2012;33:545-49

20. Albers GW, Thijs VN, Wechsler L, et al, for the DEFUSE Investigators. Magnetic resonance imaging profiles predict clinical response to early reperfusion: the diffusion and perfusion imaging evaluation for understanding stroke evolution (DEFUSE) study. Ann Neurol 2006;60:508-17

21. Nagakane Y, Christensen S, Brekenfeld C, et al. Epithet: positive result after reanalysis using baseline diffusion-weighted imaging/perfusion-weighted imaging co-registration. Stroke 2011;42:59-64

22. Hopyan J, Ciarallo A, Dowlatshahi D, et al. Certainty of stroke diagnosis: incremental benefit with CT perfusion over noncontrast CT and CT angiography. Radiology 2010;255:142-53

23. Pettersen J, Aviv R, Black S, et al. Global hemispheric CT hypoperfusion may differentiate headache with associated neurological deficits and lymphocytosis from acute stroke. Stroke 2008;39:492-93

24. Hopyan JJ, Gladstone DJ, Mallia G, et al. Renal safety of CT angiography and perfusion imaging in the emergency evaluation of acute stroke. AJNR Am J Neuroradiol 2008;29:1827-30

25. Krol AL, Dzialowski I, Roy J, et al. Incidence of radiocontrast nephropathy in patients undergoing acute stroke computed tomography angiography. Stroke 2007;38:2364-66

26. Lima FO, Lev MH, Levy RA, et al. Functional contrast-enhanced CT for evaluation of acute ischemic stroke does not increase the risk of contrast-induced nephropathy. AJNR Am J Neuroradiol 2010;31:817-21

27. Oleinik A, Romero JM, Schwab K. CT angiography for intracerebral hemorrhage does not increase risk of acute nephropathy. Stroke 2009;40:2393-97
28. Tippins RB, Torres WE, Baumgartner BR, et al. Are screening serum creatinine levels necessary prior to outpatient CT examinations? Radiology 2000;216: 481-84

29. Olsen JC, Salomon B. Utility of the creatinine prior to intravenous contrast studies in the emergency department. J Emerg Med 1996;14:543-46

30. Aulicky P, Mikulík R, Goldemund D, et al. Safety of performing CT angiography in stroke patients treated with intravenous thrombolysis. J Neurol Neurosurg Psychiatry 2010;81:783-87

31. Mnyusiwalla A, Aviv RI, Symons SP. Radiation dose from multidetector row CT imaging for acute stroke. Neuroradiology 2009;51:635-40

32. Jackson D, Earnshaw SR, Farkouh R, et al. Cost-effectiveness of CT-perfusion for selecting patients for intravenous thrombolysis: a US hospital perspective. AJNR Am J Neuroradiol 2010;31:1669-74

33. Young KC, Jahromi BS, Singh MJ, et al. Hospital resource use following carotid endarterectomy in 2006: analysis of the nationwide inpatient sample. J Stroke Cerebrovasc Dis 2010;19:458-64

A.J. Fox

S.P. Symons

P. Howard

R. Yeung

R.I. Aviv

Neuroradiology Division

Department of Medical Imaging

Sunnybrook Health Sciences Centre

University of Toronto

Toronto, Canada

http://dx.doi.org/10.3174/ajnr.A3099 